\title{
Exploring and Designing for Memory Impairments in Depression
}

\author{
Chengcheng Qu \\ School of Computing and \\ Communications \\ Lancaster University \\ c.qu3@lancaster.ac.uk
}

\author{
Corina Sas \\ School of Computing and \\ Communications \\ Lancaster University \\ c.sas@lancaster.ac.uk
}

\author{
Gavin Doherty \\ School of Computer Science and \\ Statistics \\ Trinity College Dublin \\ Gavin.Doherty@tcd.ie
}

\begin{abstract}
Depression is an affective disorder with distinctive autobiographical memory impairments, including negative bias, overgeneralization and reduced positivity. Several clinical therapies address these impairments, and there is an opportunity to develop new supports for treatment by considering depression-associated memory impairments within design. We report on interviews with ten experts in treating depression, with expertise in both neuropsychology and cognitive behavioral therapies. The interviews explore approaches for addressing each of these memory impairments. We found consistent use of positive memories for treating all memory impairments, the challenge of direct retrieval, and the need to support the experience of positive memories. Our contributions aim to sensitize HCI researchers to the limitations of memory technologies, broaden their awareness of memory impairments beyond episodic memory recall, and inspire them to engage with this less explored design space. Our findings open up new design opportunities for memory technologies for depression, including positive memory banks for active encoding and selective retrieval, novel cues for supporting generative retrieval, and novel interfaces to strengthen the reliving of positive memories.
\end{abstract}

\section{CCS CONCEPTS}

- Human-centered computing • Human-centered interaction (HCI) - Empirical studies in HCI

\section{KEYWORDS}

Depression; memory impairment; memory technologies; cues

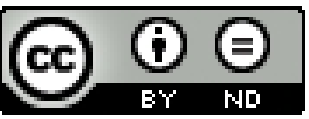

This work is licensed under a Creative Commons Attribution-NoDerivs International 4.0 License.

(C) 2019 Copyright is held by the owner/author(s)

ACM ISBN 978-1-4503-5970-2/19/05.

DOI: https://doi.org/10.1145/3290605.3300740
ACM Reference format:

Chengcheng Qu, Corina Sas, Gavin Doherty. 2019. Exploring and Designing for Memory Impairments in Depression. In 2019 CHI Conference on Human Factors in Computing Systems Proceedings (CHI 2019), May 4-9, 2019, Glasgow, Scotland, UK. ACM, New York, NY, USA. Paper 510, 14 pages. https://doi.org/10.1145/3290605.3300740.

\section{INTRODUCTION}

Depression is a condition with significant social costs which raises significant challenges both for interventions used in clinical settings and for support provided in daily life. While depression is primarily known for the dysregulation of affect, it is also characterized by cognitive dysfunction including memory impairments such as negative bias, overgeneralization and reduced positivity [13,25].

While most HCI research on memory technologies has focused on episodic memory impairments such as those associated with dementia, less work has focused on building technologies for autobiographical memory impairments to help people living with other mental disorders such as depression. Memory impairments in depression are however fundamentally different; their effect is felt not through the loss of episodic memories, but rather difficulties in the retrieval of episodic memories through higher levels of autobiographical memories such as general events and lifetime periods. Another distinct body of work explored computerized interventions for depression such as online Cognitive Behavioral Therapy (CBT). Such interventions address memory impairment, not as the main focus, but rather through a subset of psycho-educational materials concerned with negative thinking patterns, and tools for tracking mood $[9,10,17]$, which provide limited support for the distinctive autobiographical memory impairments associated with depression. Our work aims to bridge these two strands of work to contribute to the design of novel classes of technologies that specifically address memory impairments in depression. We argue that understanding the specific memory impairments in depression, and the new range of challenges they pose, offers a rich opportunity to extend $\mathrm{HCI}$ research on 
memory technologies in new directions. This paper is an initial step towards exploring this space and focuses on the following research questions:

1. How are memory impairments in depression addressed through tailored interventions used in clinical and neuropsychological practice?

2. What is the role of materials in these memory interventions and how are they employed in therapeutic practice?

3. How can therapeutic memory interventions for depression inform the design of novel memory technologies?

To address these questions, we report on interviews with ten experts working in clinical and neuropsychological practice with people living with depression. This includes four neuropsychologists, for whom memory impairments will form a larger part of treatment, as well as therapists from other backgrounds, for whom memory impairments will form part of a wider treatment strategy. The main contributions of this work include a richer understanding of how memory impairments in depression are addressed in clinical practice and design guidelines for new memory technologies for people living with depression. The paper's contribution is threefold: (i) sensitizing HCI researchers to the limitations of current memory technologies, (ii) broadening their awareness of memory impairments beyond those associated with episodic memory recall, i.e., unlike ageing or dementia, other conditions such as depression or PTSD do not involve impairments of episodic memory recall [60], (iii) inspiring $\mathrm{HCI}$ researchers to engage with this less explored design space for developing novel classes of memory technologies to address alternative memory impairments such as the ones in depression.

\section{LITERATURE REVIEW}

\subsection{HCI Work in Depression Treatments}

The last decade has witnessed a massive growth of $\mathrm{HCI}$ work on affective health [48]. In particular, HCI work on depression has considered a range of systems aiming to support detection of depressive symptoms, management of short-term negative emotions, tracking of depressive symptoms, and online management of depressive symptoms over the long term. Work on diagnosing depressive symptoms has focused on analyzing online daily activities (e.g., Reddit [3,6], Twitter [5,58], Instagram [37]) to detect depression (e.g. postpartum changes [5], suicidal ideation [6], self-disclosure [3], severity of depression [58], and mental health issues in general [37]). Another strand of relevant $\mathrm{HCI}$ work has looked into interactive devices for guiding mindful practices [14] to reduce high-intensity negative emotions in the short-term [4] or tracking depressive symptoms [38]. This body of work builds on positive psychology [29], utilizing lifelogging technologies [29] to better support coping strategies through expression [43] or increased social communication [61]. Retrospective self-tracking is itself subject to a number of sources of bias [18].

In addition to tracking, the design of computerized psychological treatments for depression has been explored by HCI research [10]. Such interventions tend to include psychoeducation sessions, requiring users to complete a number of achievable goals [9]. These online mental treatments are often based on highly structured and goaloriented psychological treatments such as CBT or Solution Focused Therapy (SFT) [9]. It has been argued that the structured nature of the content of these interventions, and the goal-oriented user journey make them more amenable to delivery via computer [10]. However it is important to also note that at present CBT is one of the most evidencebased and widely used approaches in both the clinical context and in internet-delivered therapy [10,17,47] and is currently as one of the first-line treatment options for mild to moderate depression, especially in the UK [17].

An important challenge for e-Health programs such as online CBT is the rate of attrition [20]. To improve user engagement, Doherty and colleagues [17] suggested a number of strategies including delivering more personalized and multimodal content, such as by allowing users to tailor their preferred treatment path. Accessing information through other modalities such as video [9], games [2,9,11], or conversation with embodied agents [22] has also been suggested, especially to increase young people's engagement with their treatment for depression.

While current computerized depression treatments are primarily based on highly-structured interventions, other unexplored possibilities include free-form interventions which build on people's life-narratives [9]. Coyle and colleagues [10] suggest approaches which focus specifically on clients' sense of self, and how they construct the narrative of their life stories with the aim of changing thinking patterns to alleviate depression. In addition, it has been suggested that the design of computer-aided systems for clinical treatment should support a broad range of therapists, treatment settings, and clients [9]; there is thus an opportunity to support a range of person-focused therapies by addressing common factors in depression.

Another challenge faced in the design of computerized systems is that as the treatment is associated with their 
illness, users may stop using the system after they feel like their depressive symptoms have improved [17]. However, quitting treatment early may mean that some cognitive impairments remain and raise the risk of relapse [13]. An interesting possibility is to investigate solutions, which can be used inside or outside of treatment.

All the above issues motivate the exploration of new technologies for the treatment of depression, with less focus on linearly-delivered training content, and less dependence on text and reading skills. This requires that the technology involve more of the client's self-relevant materials and have a less explicit framing as "treatment for depression", but instead aim to raise the resilience of people at risk of depression.

\subsection{HCI Work in Memory Technologies}

Memory technologies have mostly focused on addressing episodic memory loss within non-clinical populations or of people with dementia. In particular, lifelogging technologies capture episodic memory to support later retrieval [26,52], reminiscence $[16,51,53,56]$ or mood regulation [31]. HCI work supporting memory in dementia $[1,24,35,36,50]$ has explored lifelogging technologies such as SenseCam to capture episodic memories and support episodic retrieval through the autobiographical memory system [49].

2.2.1 Systems supporting capturing and organizing cues for EM. Episodic Memory (EM) contains highly detailed sensorial information regarding specific life events, which can either decay quickly or become integrated within the autobiographical memory system from where they can be vividly retrieved [7]. With the help of modern memory technologies (such as contextual sensors and massive digital storage techniques [54]), people can record more and more sensorial memory cues (e.g., visual cues with camera $[26,30,52]$, or verbal cues with microphones [16]) to support episodic memory in everyday life. However, such technologies can generate a huge number of memory cues, which are impractical for users to review and directly make sense of (e.g., 1500 images a day [30]). Thus, Sellen and colleagues [54] suggest selectively capturing episodic events for addressing particular memory impairments. Some researchers suggest filtering valuable memories at the moment of capture, either automatically (e.g., by filtering memories with high emotional arousal [52], or via user selection (e.g., self-crafted memory cues [50]).

As episodic memories cues accumulate over time, a framework is required to organize and represent captured cues. Recording captured memory cues and constructing them in a narrative representation is a method that works well with users' preferences and natural habits [44]. Such work includes narrative diaries of everyday events, modalities of cues include video summaries or animations [30], bio-sensing data [55], or combined multimedia memory cues [42].

2.2.2 Systems supporting cue-based remembering through $A M$. Autobiographical memory (AM) is the "remembered self" across the lifespan, which provides a long-term framework for episodic memories. Current memory technologies predominately support user needs in directly accessing episodic memories through the AM system, by representing memory cues that explicitly target specific episodic memories [26]. Such supports in direct retrieval could benefit people with memory loss problems, to reconstruct forgotten memories for later reminiscence [56], memory sharing [32], emotion regulation [31], or alleviating dementia [35]. However, presenting users with captured memory cues does not necessarily mean they can remember successfully. The captured cues are just collections of data for assisting the remembering process, but not the process itself. As stated in [27], remembering is a process of re-construction within the self-memory system and retrieving specific memories based on users' current beliefs. The retrieved memories will always be altered by people's current dominant self-beliefs, and their current interpretation of the memory. Van den Hoven and colleagues [27] further suggested that AM technologies should be flexible in presenting memory cues for supporting memory retrieval.

We see from the above that work on memory technologies has mainly focused on episodic memory impairments (i.e., memory loss in dementia or forgetting). $\mathrm{Qu}$ and Sas [45] have started to introduce autobiographical memory training for depression to the field of HCI and called for future work in advancing memory technologies, however, HCI research has not widely addressed several important aspects of autobiographical memory impairment, and in particular how people's dynamically-changing sense of self can distort their ability to remember. These complex impairments arise in a variety of mental conditions, including autobiographical memory impairments in depression, and flashback trauma memories in PTSD.

\subsection{Memory Impairments in Depression and Memory- Based Interventions}

2.3.1 Autobiographical memory and self-identity. Retrieval through the Autobiographical Memory System (AMS) involves the autobiographical knowledge-base and people's self-identity [8]. The former contains memories across the lifespan at three levels of abstraction (Fig.1): lifetime 
periods (highly semantic), general events (part semantic, part episodic), and episodic memories [7,8] while the working-self [8] involves information about self-identity, and is critical for accessing each episodic memory. The AMS supports two modes of retrieval: generative retrieval and direct retrieval. Generative retrieval is a memory searching process involving one's self-identity. As shown in Figure 1, the generative memory-searching process starts with a cue activating the retrieval mode, accessing and evaluating memories at all three memory levels, and elaborating the search-cue iteratively, until a matched specific memory is reached. Direct retrieval is triggered through cues with direct links to specific episodic memory, with less involvement of self-identity.

Consistent findings in psychology have shown that people living with depression have three distinctive AM impairments (Figure 1): negative bias, overgeneralization, and reduced positivity [13,25]. These memory impairments mainly disrupt the generative retrieval process. Several memory-based interventions [19,34,46,57] have been designed to address such memory impairments in depression [25].

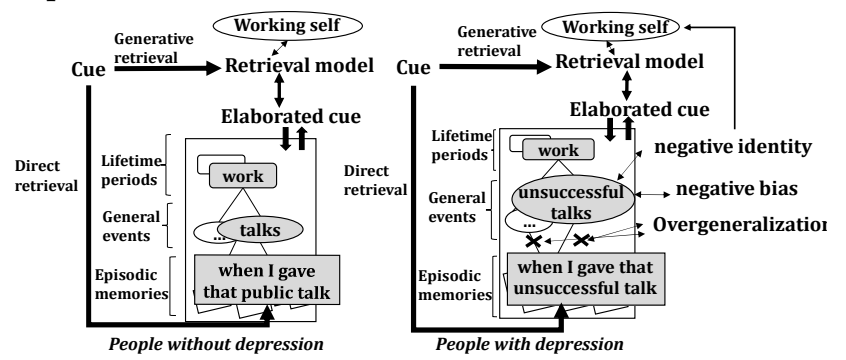

Figure 1. Memory retrieval in people without depression (left), and with depression (right)

2.3.2 Three memory impairments in depression and targeting interventions. Psychological work has identified three memory impairments in depression [13]: negative bias, overgeneralization, and reduced positivity and four main tailored interventions [25]. Concreteness Training (CNT) trains people's ability to look back at specific events in a concrete manner to reduce negative bias and overgeneralization. Memory specificity training (MEST) trains people's ability to retrieve episodic memories to address overgeneralization. Competitive Memory Training (COMET) trains the ability to retrieve positive memories for building a positive identity for addressing negative bias and reduced positivity. Cognitive Bias Modification-Imagery (CBM-I) trains people's ability to cope and practice positive interpretation through imagination for addressing reduced positivity.
Negative Bias [13]. Research has shown [13] that people with depression tend to have negative self-identity, which contributes to negative bias. They tend to pay attention to negative materials or to interpret vague scenarios in a negative manner, which leads to the retrieval of a larger number of negative memories compared to the non-depressed population. This process strengthens the access path of negative memories from the memory system making negative memories easier to retrieve and positive ones harder to retrieve. As time goes by, the imbalanced remembered self (AM) continues to fuel a stronger negative self-identity. Several memory interventions have been developed to address negative bias [25], including COMET [34] and CNT [19]. While COMET helps clients to construct a positive self-image to guard against the negative one, CNT helps depressed clients to disengage with their retrieved negative memories by analysing them in a concrete manner.

Over-generalization [12,13]. People living with depression have difficulties in accessing both specific episodic memories, and their details through the generative retrieval process. As shown in Figure 1, the memory search process starts from accessing the higher levels of AMS such as lifetime periods and general events. Unlike nondepressed individuals, people with depression tend to finish the search process before reaching the episodic memory level. In addition, as mainly negative memories could be retrieved due to negative-bias, the retrieved abstract, negative memories further contribute to an abstract and negative life summary; "My life is a total failure". People with depression do not have problems with the direct retrieval of episodic memories, as their memories are not lost, but simply harder to access [59]. As described in [25], MEST [13,46] and CNT [19] are interventions addressing overgeneralization. MEST helps users enhance specific memory retrieval with emotional word-based cues, while CNT help them retrieve details in their specific negative memories and analyze them in a more concrete manner.

Reduced Positivity [13]. Due to their negative selfimage, positive memories of people with depression are less vivid and emotionally-intensive, so they contribute little to users' mood even when retrieved successfully [13]. In addition, as depression draws attention to negative material, fostering rumination and negative mood, it can also contribute to worsening of mood while reviewing positive memories. Apart from clinically depressed people, healthy people with low mood [33], or history of depression [15] also face this risk. As suggested in [25], COMET [34] helps users build positive self-image by repeatedly imaging 
positive experiences or retrieving positive memories, while CBM-I [57] helps users to practice positive interpretation of neutral scenarios.

These three memory impairments, as indicators of depression, may persist even after a depressive episode is resolved, contributing to the next one [13].

\section{METHODOLOGY}

We conducted semi-structured interviews with ten experts in clinical or neuropsychological practice, with at least six years of experience of working with people living with depression. The study aimed to explore their practices of addressing memory impairments in depression. Participants (eight female, two male) have on average 15 years of experience (range 6-30) in working with their clients living with depression. Seven participants have expertise in CBT (P1, P2, P3, P4, P8, P10), two are neuropsychologists with expertise in memory training in depression (P5, P9), and two have expertise in both CBT and memory training in depression (MEST) (P6, P7). Five participants have Ph.D. degrees in Psychology, four have postgraduate degrees (MA, certificate, diploma) in psychotherapy, while another one has a BSc degree in psychology. Six participants were from the UK, while the other four were from Belgium and the Netherlands.

Participants were recruited through email advertisements from CBT associations and an online database of clinical practitioners and researchers. The semistructured interviews were carried out individually through phone or Skype calls. During the interviews, we asked participants about their practice and how they addressed memory impairments in depression. We also asked about their challenges in conducting the treatments as well as engaging treatment-recipients. Interviews lasted about an hour, were audio recorded and fully transcribed.

Data analysis involved hybrid coding which ensured that themes were partly grounded in the literature and partly derived from our findings, offering the advantage of balancing the knowledge of existing theory with the sensitivity to theoretical novelty. The first author completed the interviews and the initial coding. Questions in the interview include: (regarding overgeneralization) "Have you worked with clients with overgeneralized memories?", "Have you tried to address this overgeneralization problem, if so, how?" This strategy was part of the hybrid approach [21], integrating the framework from the literature with accounts of the work practices of individual therapists.

Data analysis took over three months, engaging all authors through weekly meetings where the codes with quotes were shared, discussed, and iteratively revised until agreement was reached regarding the final list of codes and their definitions. A conceptual framework developed from literature supported deductive coding, i.e., impairments such as overgeneralization, negative bias, reduced positivity, or memory processes such as encoding or retrieving. These were refined as new codes emerged, i.e., approaches such as encoding positive memories or retrieving negative memories.

\section{FINDINGS}

In this section, we discuss the clinical approaches used by participants to address the three key areas of negative bias, overgeneralization, and reduced positivity. We report them together with what type of memory (i.e., negative or positive) these approaches apply to, as well as which memory processing stages are targeted by each approach. We then conclude this section by reviewing specific materials that these clinicians used in their practice to support people managing depression.

\subsection{Approaches Addressing Negative Bias}

Negative bias refers to the pervasive negative-tone of depressed individuals' life-narrative, which is due to their preference for paying attention to negative materials and negative interpretation. People living with depression also tend to retrieve negative memories much faster and easier compared to positive ones $[13,25]$. Negative memories with high-accessibility (and positive memories with lowaccessibility) further enhance the negative perspective of their life-stories as well as increasing the likelihood of rumination.

We identified several approaches from both clinical and neuropsychological practices to address the above problems, including reducing the negative effects of retrieved negative memories and increasing clients' ability to encode and retrieve positive memories.

4.1.1 Analyze and learn from negative memories. Both CBT therapists and neuropsychologists help their clients to analyze negative memories to support disengagement. These approaches aim to make negative memories less negative, and their emotional meaning easier to be disengaged from in future retrieval. The quotes provided in this section are illustrative of the common themes identified. Findings suggest two ways of supporting analysis and reflection on negative memories. Some therapists directly inform their clients about their problematic thinking style, P8 (CBT therapist): "the thinking errors [could be] jumping to conclusions, thinking more negatively towards yourself and other people, or you are 
harsher on yourself then you are to other people". Another approach is to provide detailed scaffolding questions for guiding clients in analyzing and learning from their retrieved negative memories, $\mathrm{P} 1$ (CBT therapist): "they need to learn what is it that creating the negative experience? What is that about? Is that about the type of people they attract? Is it something about them, which causes these things to go wrong? They will need to explore that, and then they might do differently in the future". This quote provides evidence of therapists' effort to encourage identification of unhealthy thinking patterns, to support disengagement from negative memories, and future healthier emotional or behavioral responses.

4.1.2 Reframe clients' negative interpretation and emotional response and encode reframed memory. Another approach for reducing the negative effects of negative memories is found in both clinical and neuropsychological practices. P5 (neuropsychologist) gave an example of a possible approach based on the Cognitive Bias Modification methodology [57]: "for example, imagine 'a cup of coffee behind a rainy window'. The depressed persons' standard interpretation would be 'I feel isolated', but the alternative interpretation would be: 'it is a lovely afternoon, we are enjoying a lovely cup of coffee'. So you [could] expose clients to this kind of negative [mental] images, and then align them to interpret the meaning of these images [and reframe them more positively]". We also found similar approaches for reducing negative-biased interpretation through reframing negative memories mentioned by P1: "a client [was] upset because [her] friend had a job she was also applying for, and she thinks it is unfair. However, I prompted her by saying: 'okay you told me that you are upset and worried about this, but is there another way of viewing it?' That could offer them [options to reframe their interpretation] and view the situation differently; [so that they could] reframe their emotion response and feel more positive about it".

The idea of this reframing process is to help clients to both reframe their memories less negatively and to encode the reframed memories in their memory system. As these negative memories could be less negative if retrieved in the future, depressed clients could disengage from these memories more quickly and easily over time.

4.1.3 Encourage switching attention from rumination to retrieved memory itself. Depressed individuals have a higher tendency to avoid retrieving details of their memories and rather distract themselves through rumination [13]. An approach for addressing this problem was mentioned mostly by neuropsychologists delivering a memory-based intervention (MEST) [46]. Three neuropsychologists (P5, P6,
P8) mentioned their approach in guiding the clients to focus on the memory itself until it is successfully retrieved with as many concrete details as possible. For example, P7 (clinical MEST therapist): "sometimes clients do start to ruminate about their negative memories. Then [as a therapist], you say, 'OK, I guess it's very difficult for you. However, just think about this family dinner and let us see what details are in there'. Therefore, you distract them from 'thinking' about the memory, by retrieving details of that memory". This illustrates an approach for disengaging from rumination by encouraging clients to fully engage with the memory retrieval instead of allowing their attention and cognitive resources to engage in rumination.

The low-accessibility of positive memories for depressed individuals also contributes to a negative-biased and unbalanced life-perspective. Therapists thus suggest building a stock of positive memories to help and encourage clients to encode positive memories over time, as a process of enriching the collection of retrievable positive memories. Such positive memory banks could also help users to increase their positive memory retrieval and thus create a balanced view of life-perspective. We have identified three strategies based upon positive memories:

4.1.4 Encourage active behaviors for encoding positive memories. If clients cannot draw from a rich collection of positive memories, therapists would bypass the choice altogether by creating opportunities for positive events and encouraging their purposeful encoding. For example, within the treatment, several therapists deliberately introduce specific activities such as arts and crafts that are likely to induce positive emotions and encourage clients to actively encode them as positive memories. P2 has mentioned her approach as: "I help them trying to retrieve the positive memory of the treatment [...] we are trying to get (clients) to realize that they are making gains right now". The above findings suggest opening up new design opportunities to better support such cue creation. P10, a CBT therapist, has also mentioned: "I guide users to create artworks in the treatment, so my clients can take them back home and retrieve the happy moments in the treatment". This quote indicates the important value of a tangible cue of a positive activity, which can trigger later positive memory retrieval.

While positive memories can be created as part of the treatment, they can also be made within clients' everyday life, since negative bias impairs depressed individuals' abilities to pay attention to positive materials. P2 describes this process as: "(they feel like) their whole life is always negative, it's hard for them to realize that positive things can happen, or even that positive things are happening currently". 
CBT therapists thus encourage their clients to increase their ability to be present and pay attention to current positive events and to encode such memories in specific detail for easier later retrieval. For example, P3 (CBT therapist) mentioned: "[I use techniques, [for] pointing out things that [clients] are grateful for in the present moment, or things they do that nurture them, to balance the things to displease them". P3 encourages clients to take notice and encode all daily emotional events: "encouraging clients to notice positive things happening in their life, unpleasant things as well as the difficulties, could tackle their overgeneralized autobiographical memories".

4.1.5 Encourage positive memory retrieval to disengage from rumination. We found a prevalence of this approach in both CBT therapies and neuropsychological treatments. For example, P4 has mentioned his approach of using clients' positive memories to help them disengage from rumination: "I use his past successes [to help him] feel balance and distract him from his pre-worry about his physical health". P6, as a neuropsychologist with background in CBT, also suggested the importance of having available positive experiences for depressed clients to engage with and to distract from negative thinking: "the most important thing for clients to shift their bad habits of negative [ruminative] self-thinking, thinking [...is to] have enough positive collectible experiences where they do manage to disengage from that ruminative thinking and to experience, [and] shift their attention or focus to something else". An interesting outcome is that although these memories could be cued through modalities other than words, findings indicate limited visual or tangible materials being used to facilitate positive memory retrieval.

4.1.6 Encourage positive memory retrieval to create a balanced view. Therapists (both neuropsychologist and CBT therapists) further mentioned the importance of a positive memory bank for increasing clients' ability to retrieve such memories and to build a more balanced view of life. For example, P5 (neuropsychologist) suggested: "[positive memory bank is useful], as they [clients] can thus look back and say 'well no, I am not always a failure in my life, not everyone in my relationships rejected me' ". P2 (CBT therapist), has also agreed the value of a positive memory bank in promoting balanced life-review: "[addressing negative bias is about] get them to see balance, challenge their negative thoughts, so [...] having them describe the situations in the past that have gone better is more powerful than just say 'everything will be alright' ".

Other CBT therapists also suggested a positive memory bank by providing illustrative examples of its value, P1: "I let them feel balance about their life, so they feel like 'something good happened' [...] I do have the capability to have good experience in a relationship [...] because it had happened". P4 also mentioned using positive memories as a counterexample to break clients' negative life perspective: "I pick out some of the [past] successes [...] so he was able to acknowledge [that he is not a failure as he thinks]". P5 further suggested that such a positive memory bank could help clients to practice and improve their abilities in positive memory retrieval: "this kind of bank of memories, with much enriched contextual details, could help [them] to increase (the accessibility of) these memories with creating more memory traces [for retrieving them]".

\subsection{Approaches Addressing Overgeneralization}

People living with depression not only tend to retrieve their past in a negative manner, but also do so in an overgeneral style. Overgeneralized memory, as a cognitive marker of depression, refers to the cognitive dysfunction affecting the retrieval of episodic memories and their details $[12,13,46]$. Negative bias and overgeneralization reinforce each other and contribute to a profound negative life summary and self-perception (an example given by $\mathrm{P} 4$ : " $m y$ whole life I have struggled') and of the world as in the example provided by $\mathrm{P} 1$ : "all my relationships failed".

4.2.1 Training clients' ability to retrieve specific memories through the generative retrieval process. This approach is mainly applied in neuropsychological treatment. Neuropsychological interventions [25,46] aim to reduce negative overgeneralization by training clients' ability to retrieve episodic memories through the generative retrieval process (section 2.3). Training the generative retrieval process consists of supporting clients to move from highlevel autobiographical memories such as lifetime periods, and general events towards retrieving specific-level episodic memories. Generative retrieval is mostly prompted by what we identify to be new types of cues: generic, and word-based. For example, in neuro-psychological memorybased treatment (MEST [46]), clients practice memory retrieval with emotional word-based cues, such as "successful" or "disappointed". Such cues should be generic rather than specific to ensure that only the generative retrieving process is activated, rather than direct retrieval usually cued by photos. Cues also have to involve certain emotional valence (neutral, negative, positive), as depressed people experience more severe difficulty in memorysearching from emotional cues [46]. As described in the MEST protocol, MEST therapists trigger clients' memory retrieval through standardized word-based cues, such as "enthusiastic" (positive cues), "telephone" (neutral cues), "stressed" (negative cues). Once clients come up with a memory (the first attempts are normally not specific 
enough), therapists then guide them to retrieve more specific details through scaffolding questions, until a specific episodic memory (normally an event that has lasted for less than 24 hours) is retrieved. We consider further this scaffolding process below.

4.2.2 Providing scaffolding to help generative retrieval. This approach is usually takes place in MEST training. It aims to scaffold clients' generative retrieval process and help them address their inability to access episodic memories. For example, P6 (neuropsychologist specialist in MEST): "when the client starts with 'I am never happy', [then I will say] 'Can you think of a time when you felt happy in the past?' 'When I went on vacation' 'OK that's a little more specific' [so this is how] you help your clients to work through that hierarchy: from vacation to the trip to the seacoast, and then to a specific trip to the seacoast which lasts a week. Then I ask and challenge the client to come up with a specific day or event or moment in that one-week trip to the seacoast”. P6 also suggests leveraging social support particularly though group intervention "the nice thing [of working in a group] is that not only [the] trainer is challenging the clients, the clients are also challenging each other".

4.2.3 Training abilities in retrieving details of specific episodic memories. This was mentioned by neuropsychologists as focusing on in-depth specific memory retrieval after their clients identified and retrieved a specific, episodic memory successfully, P6 "when [clients] come up with specific memories, we challenge them to come up with extra contextual sensory perspectival details, with their specific memories”. One neuropsychologist further provides suggestions of how she would use such a guiding process within a mobile application: "[The app would] train participants to retrieve positive events and retrieve them fully detailed, by asking users to describe details of those events: normally at least 15 details”. P8, as a CBT therapist utilizing behavior activation who also acknowledged the value of MEST training, has put effort in integrating MEST elements into her CBT treatment. In this respect, she suggested positive immersion in the retrieved memory, and its specific details: "[...] close your eyes, and imagine this [experience], imagine it, and pause with this, and feel [...] I expose them [clients] to their positive feelings, make them open up and let these feelings enter their system".

4.2.4 Encourage retrieving positive memories to break negative thinking style. This approach was specifically found in CBT therapies in training overgeneralization (more than half of the interviewed CBT experts). As in treating depression, traditional clinical treatments such as CBT emphasize changing negative thinking patterns due to negative-biased overgeneralization, therapists thus predominately address overgeneralization by driving clients directly to specific positive memories. They also use positive memories that are retrieved by the clients as counterexamples for breaking the over-generalized thinking style and negative life summary. Findings indicate two ways of triggering positive memory retrieval. Some CBT therapists trigger it with simple questions by directly challenging clients' negative thinking, P2: "really? Don't you have any positive memories? Is that true?" or P1: "so are you saying that you never get enjoyment out of doing things?" These quotes illustrate therapists' efforts to make clients realize the problem of their negative, overgeneralized thinking, and start retrieving positive memories. The second method for triggering positive memory retrieval consists of guiding the clients to retrieve specific life events that are generally enjoyable, P1: "maybe [...] there was a time that you have experienced a good relationship?", or bring awareness to such positive memories as reflected directly in clients' accounts during the treatment, P8: "when he was describing what happened at work when I heard something good [...] I stopped him". CBT therapists also mentioned the value of knowing clients' background as a way to better prompt this retrieval process, P2: "[if I know their background well] I would say 'what about the day when you got married?' or 'How about the day when your kids were born?'”.

\subsection{Approaches Addressing Reduced Positivity}

As suggested by [13], even if depressed individuals can successfully retrieve a positive memory, such memories are less vivid and emotionally-intensive, impeding depressed individuals from experiencing the positive emotions associated with it. We report three main strategies identified for addressing this problem.

4.3.1 Helping reconstruct, visualize, and retrieve associated positive emotions of positive memories. Both neuropsychologists and CBT therapists mentioned this approach. It consists of first helping clients to retrieve many details of specific positive memories, P9: "just think of an event [...] imagine it including some of the details, for example [...] what happened exactly, [...] as well as how they were feeling [...], in 5 to 15 details". The second step is to help clients through scaffolding questions to retrieve positive emotions associated with these memories, otherwise, as P8, a CBT therapist described, their clients would just "jump over it". This step focuses on positive emotions, as such memories are important in helping depressed individuals to experience rather than avoid 
specific memory details [13], P8: "so it is using the image vividly, and feel what he [client] feels at this moment. To open up and to let it enter the system". The most used scaffolding questions include, P8: "when I heard something good, [like a compliment or nice interaction] I stopped him and instead of [let him] just going over it, I asked him to do some imagery [and] stand still to feel the feelings or imagine the situation again". P8 further illustrated details of her scaffolding questions to support reliving this memory: "[I] say 'close your eyes, and imagine this experience. Imagine it and, pause with this, and feel.' So [the process] is using the image vividly and feel what he feels at this moment. To open up, and to let it enter the system". This quote illustrates the value of slowing down and paying attention to a positive memory, which opens up new design opportunities supporting a rich reliving process.

4.3.2 Enhancing retrieved positive memories with a positive cognition model, and help encoding reframed positive memories. This approach was suggested by a CBT therapist, P3. She reported her efforts to enhance these positive memories by integrating a positive cognition model with these memories [23], helping the clients to encode enhanced positive memories in their memory system: "[I] encourage users to encode their memory holistically. Get clients retrieving things that they are grateful for [...] and encourage them to use the CBT model to break these moments down, create an emotional memory [that] involves emotion, cognition. So whenever you retrieve this positive memory, not only you will retrieve the memory itself, you will also retrieve the full [CBT] model behind it”. As indicated above, this approach aims to lead clients to repeatedly retrieve their positive memories to encode future positive memories in a holistic manner.

4.3.3 Utilizing positive memories to build better selfidentity. More than half of the interviewed participants mentioned the importance of boosting users' self-identity. Some therapists specifically identified their approaches of utilizing positive memories for this purpose, P1: "I use that [positive memories provided by my clients] to help them feel a bit more confident about themselves, like, "you know, you do have the capability to have good experiences in a relationship [...] because it had happened once". However, such an approach for boosting self-identity is currently supported only by verbal conversations, with limited efforts focused on capturing these powerful positive memories linked to one's sense of identity.

We argue that purposefully encoding these emotional events as they occur during the therapeutic session can later provide valuable cues for key positive memories weaved in one's sense of self.

\subsection{Clinical Usage of Materials}

Study findings indicate that current depression treatments are predominantly based on conversations. Some therapists use standardized materials in their treatments, such as printed paper of the $\mathrm{ABC}$ worksheet [62] and feelings wheel [63] in CBT (P2, P3) or word-based cues in MEST training (P6, P7). Other therapists select materials that may cue their clients' positive past experiences. For example, P4 uses songs and visual objects to remind his clients of significant life events, integrating materials that are relevant to each specific client's background. In most case, therapists are the ones to provide training materials, but occasionally clients' written diaries are also employed as part of the treatment (P3, CBT and P6, P7, MEST).

4.4.1 Self-relevant materials. P4 specifically mentioned the value of self-relevant materials, for example, as visual cues: "things that could trigger a retrieval, like photograph albums [...] an old dictionary or an old textbook. (They could) unlock other memories [...] Once I used some normal computer magazines from the 1990s, because a client was working in the IT industry [during that time], [it's the type of magazine that] you could have bought in any stores". P4 also utilizes the audio cues such as songs: "you could almost consider that as a signature. These are a personalized set of stimuli or triggers [One time I played an old song in front of my client] when the music came, because they are her brothers' favorite artist, she suddenly felt herself upset [for feeling the time spent with him] as quickly as it started." From a CBT perspective (P4), that's: "something that is worth the grasp and makes her reflect on this retrieval". These quotes illustrate the importance of vivid visual and audio cues, as specific memory cues could trigger rich sensorial direct retrieval [8,27]. One concern is that such sensorial memories could worsen clients' mood with potential harmful impact, and care should be taken in designing and using them with clients.

Some CBT therapists also asked clients to write diaries, mostly on paper, as material to trigger retrieval in the next training session, P3: "and we used a lot of stories to illustrate that it's not always the case, and the diaries show what he feels for sensing”. Neuropsychologists also mentioned written diaries as important part of MEST training, P6:"the diary is just on a piece of paper, within the support group, they help each other inside [...] If they do it as homework, they will write them down, or they talk about it during the training session or in a group interventions context”. Interestingly, no participant mentioned helping their clients to collect and store these individual notes into a diary notebook for later retrieval. 
4.4.2 Standardized materials. Other self-relevant materials include self-generated personal triggers from general training materials, such as the feelings wheel [63] mentioned by P3: "(I) let clients identify triggers [such as] pleasant and unpleasant triggers. Then I get them to validate the feelings they noticed. After that, I let them realize where they identify in the body, and then get them to notice the input, and then help them to cope with the next step”. P2 also mentioned using paper-based $\mathrm{ABC}$ worksheets [62] to help her deliver cognitive behavior therapy. The idea of these standardized materials is to trigger clients' personal feelings of thoughts.

MEST [46] also uses a list of standardized word-based cues for helping clients practicing generative memory retrieval, usually from neutral word-based cues to more challenging positive word-based cues. MEST also utilizes negative cue words for training clients' cognitive flexibility in memory retrieval, especially in disengaging from rumination [39].

\section{THEORETICAL IMPLICATIONS}

We now revisit our research questions and identified limitations from prior work we reviewed earlier. We highlight the findings, especially regarding strategies and materials from current therapeutic memory interventions. We hope to inspire and inform the design of technologies to better address memory impairments in depression.

\subsection{Tailoring Depression Technologies}

As suggested in prior research [9,17], computerized depression interventions, especially online CBT platforms are mainly concerned with the delivery of pre-designed psychoeducational content. While they can incorporate a variety of more interactive exercises, these platforms currently involve limited self-relevant materials from users' life narrative. In order to fully realize the potential of technology for mental health treatment, researchers have called for more exploration of support for person-centric interventions [10]. Such an approach would involve more free-form interventions that build on users' life narratives and focus more on users' sense of self. This can include memory based interventions.

Researchers have also suggested utilizing more personalized and multimodal content to improve users' engagement [17], which further supports the inclusion of self-relevant materials.

Regarding self-relevant materials used in therapeutic memory interventions, we found that clinical approaches mainly use standardized training materials, while some therapists utilize sensorial materials to trigger emotional responses associated with certain memories. However, these specific cues may come with a potential risk of harming clients by triggering strong, negative feelings that are, described by P1: "too painful [for clients] to even talk about". Other clinicians utilize users' written diaries as part of training, but do not use them for tracking the effectiveness of the treatment.

\subsection{Tailoring Memory Technologies}

As suggested in prior research [27,54], memory technologies should be designed to strategically address users' memory impairments and support related memory functions, but we know little about how these can be achieved for people living with depression.

Previous work suggests that most memory technologies focus on supporting memory impairments at EM level by triggering direct retrieval (e.g., episodic memories, lost or forgotten $[16,26,30,35,52])$. This, however, is not a memory impairment associated with depression [59]; depression is associated with three distinctive impairments at AM level [13]. Building on this knowledge, several recently developed psychological interventions provide an interesting starting point and potential context of application for the development of new memory technologies [25]. We have presented findings on how approaches from both clinicians and neuropsychologists address each memory impairment in their work with people living with depression. We have found that: 1) For negative bias, clinicians appear to use approaches focused on improving clients' ability to retrieve both positive and negative memories, reframe negative memories, as well as encode both newly generated positive memories and reframed negative ones. 2) For addressing overgeneralization, neuropsychological approaches mostly help clients to improve their ability to retrieve episodic memories with enough specific details, while CBT therapists address negative-biased overgeneralized thinking (e.g., breaking a negative thinking pattern) by improving clients' ability to retrieve positive memories opposing their negative thinking. 3) Reduced positivity was addressed by increasing clients' ability to retrieve positive memories, fully relive and enhance them, and to encode these enhanced memories for later retrieval.

\section{DESIGN IMPLICATIONS}

We now discuss the design opportunities for addressing the identified gaps in prior research on memory and depression technologies based on the findings of this study. We will 
specifically identify the key challenges associated with each memory impairment in depression: negative bias, overgeneralization and reduced positivity.

\subsection{Positive Memory Banks for Active Encoding and Selective Retrieval}

Our findings indicate the significant and pervasive use of positive memories for addressing all memory impairments in depression. However, an important outcome is that the value of these positive memories is associated less with their retrieval and more with their active and purposeful encoding. For example, clients are encouraged to notice and record positive daily events, marking progress as their treatment progresses, as well to plan and engage in pleasurable events. Clinical practice, however, provides limited support for the capture and materialization of these memories. We can think of novel classes of memory technologies to purposefully support active encoding by identifying positive affective experiences, prompting more elaborate encoding, and rewarding the growth of these positive memories as a powerful resource to draw from in the future. Precious moments are perhaps fewer and further apart for people with depression, and hence it may be valuable to prompt their encoding. Thus, biosensor-based interfaces could offer new exciting opportunities in this space. In a critique of the total recall enabled by lifelogging, memory technology researchers have called for the importance of active curation, i.e., active process of annotating memory records [28]. With respect to depression, our findings further emphasize the importance of active or purposeful user engagement by extending it earlier in the memory process at the encoding stage.

Our findings also emphasize the importance of selective retrieval of positive memories, an aspect less recognized in HCI memory work. Previous findings have shown that retrieving positive memories can be problematic for depressed clients $[13,15]$ who at the time of retrieval are experiencing negative moods, as it can trigger rumination [41], and comparison with one's past thus worsening the present negative mood [33].

We argue for the importance of novel technologies that can adapt the retrieval of positive memories to the current emotional state of the user and particularly address rumination. For example, we can imagine technologies that prompt people to identify and retrieve positive memories as counterexamples for ruminative thoughts. In turn, this can support a more balanced perspective on life including both positive and negative experiences, and increase the accessibility and value of positive memories for breaking ruminative thinking patterns. We can also think of novel memory technologies that can address the challenge of direct retrieval which, as an effortless process, can easily be hijacked by rumination $[13,15]$.

\subsection{Novel Cues for Supporting Generative rather than Direct Retrieval}

Our review of the psychological literature indicates that memory impairments in depression disrupt the generative retrieval process $[12,13,59]$. Therefore, people living with depression may benefit more from interventions and technologies supporting generative retrieval rather than those supporting their unimpaired direct or cue-based retrieval [59]. In other words, the main challenge for people living with depression is accessing the episodic memory level within the autobiographical memory system rather than remaining stuck at the lifetime periods or general events levels. If they successfully overcome this challenge and manage to access an episodic memory, they have fewer difficulties recalling its specific details through traditional cue-based, direct retrieval. As a result, people living with depression not only benefit less from the types of cues usually explored in memory technology research, but such cues can also be counterproductive: by prompting direct retrieval these cues interrupt the generative retrieval, depriving them of the opportunity to practice the latter process (which has been shown to have positive effects in alleviating depression $[25,46]$ ).

Most technology-based cues, and in particular those captured through lifelogging technologies, record episodic events, and therefore support direct retrieval. However, people living with depression need a new type of cue to prompt generative retrieval. The main source of information we have found regarding the construction of such cues is provided by the MEST intervention where generic cues, often word-based, are used to support it. Interestingly however, we have seen therapists' efforts to augment such cues with content relevant for their individual clients such as magazines from the 1990s when the client was working in the IT industry. We argue that such important cues can in fact be supported by technology, as long as their content remains generic, i.e., about lifetime periods or general events, rather than specific. i.e., about episodic memories. For instance, we can imagine new techniques for integrating, summarizing, or visualizing technology-captured episodic cues into highlevel representations of general event or lifetime periods within which the specific episodic content remains unrecognized.

We can also imagine new techniques for identifying among one's lifelogging data ontologies of objects inferred from distinct yet related episodic events, which can be used to construct cues for generative retrieval. This would imbue 
these cues with much needed self-relevant content. Or we can think of such cues for generative retrieval as consisting of meta-data of captured emotional episodic events. The study also suggests opportunities for improving generative retrieval by augmenting these cues with scaffolding questions. One can imagine conversational agents [22] guiding memory search towards the episodic level of AMS.

\subsection{Novel Interfaces Strengthening the Reliving of Positive Episodic Memories}

We have already discussed the importance of selective retrieval of positive memories while accounting for people's current emotional states, and that in negative moods, such retrieval should be carefully leveraged. In contrast, the moments when depressed people experience neutral or positive moods can be fully leveraged for prompting retrieval of positive memories. In addition, the study indicates that such positive memories are not only difficult to access but they also are less vivid and experientially embodied. Such insights open up new design opportunities for memory technologies. We can imagine novel, rich, expressive and multisensorial interfaces that could strengthen the felt-life quality [40] during retrieval of one's positive memories. In turn, such increased reliving could further strengthen positive self-identity.

\subsection{Introducing Tailored Memory Technologies to Online Intervention Platforms}

Our previous discussion argued the importance of introducing self-relevant materials to online intervention platforms for treating depression. The study also revealed how clinicians apply self-relevant materials such as emotional memory cues within interventions. An opportunity exists for more person-centric online platforms for treating depression, which integrate memory systems specifically tailored for depression and provide materials from users' life narrative. In addition, a positive memory bank, which helps users to track positive memories, could provide a visualized training progress and a sense of achievement, and thus help increase user engagement with the intervention.

\section{CONCLUSION}

Our study explored three memory impairments in depression, namely negative bias, overgeneralization, and reduced positivity. By interviewing 10 clinical and neuropsychological experts, we identified effective approaches for addressing the challenges of these impairments; approaches grounded in both theory and clinical practice. Our findings open up new design opportunities for memory technologies tailored to the needs of people living with depression. These include positive memory banks for active encoding and selective retrieval, novel cues for supporting generative rather than direct retrieval, and novel interfaces to strengthen the reliving of positive memories.

\section{ACKNOWLEDGMENTS}

This work has been supported by AffecTech: Personal Technologies for Affective Health, Innovative Training Network funded by the H2020 Marie Skłodowska-Curie GA No 722022. The research of Gavin Doherty is supported in part by Science Foundation Ireland grant 12/CE/I2267 to the Adapt Centre.

\section{REFERENCES}

[1] Mélissa C. Allé, Liliann Manning, Jevita Potheegadoo, Romain Coutelle, Jean Marie Danion, and Fabrice Berna. 2017. Wearable Cameras Are Useful Tools to Investigate and Remediate Autobiographical Memory Impairment: A Systematic PRISMA Review. Neuropsychol. Rev. 27, 1 (2017), 81-99. DOI:https://doi.org/10.1007/s11065-016-9337-x

[2] Etaba Assigana, Eric Chang, Seungsuk Cho, Vivek Kotecha, Bing Liu, Hannah Turner, Yan Zhang, Michael G Christel, and Scott M Stevens. 2014. TF-CBT Triangle of Life: A Game to Help with Cognitive Behavioral Therapy. Proc. First ACM SIGCHI Annu. Symp. Comput. Interact. Play (2014), 9-16. DOI:https://doi.org/10.1145/2658537.2658684

[3] Sairam Balani and Munmun De Choudhury. 2015. Detecting and Characterizing Mental Health Related Self-Disclosure in Socia Media. In Proceedings of the 33rd Annual ACM Conference Extended Abstracts on Human Factors in Computing Systems - CHI EA '15, 1373-1378. DOI:https://doi.org/10.1145/2702613.2732733

[4] Matthew Barker and Janet Van der Linden. 2017. Sprite Catcher: A Handheld Self Reflection and Mindfulness Tool for Mental Healthcare. Proc. Tenth Int. Conf. Tangible, Embed. Embodied $\begin{array}{lllll}\text { Interact. } & - & \text { TEI '17 (2017), } & \text { 419-425 }\end{array}$ DOI:https://doi.org/10.1145/3024969.3025068

[5] Munmun De Choudhury, Scott Counts, and Eric Horvitz. 2013. Social media as a measurement tool of depression in populations. Proc. 5th Annu. ACM Web Sci. Conf. - WebSci '13 (2013), 47-56. DOI:https://doi.org/10.1145/2464464.2464480

[6] Munmun De Choudhury, Emre Kiciman, Mark Dredze, Glen Coppersmith, and Mrinal Kumar. 2016. Discovering Shifts to Suicidal Ideation from Mental Health Content in Social Media. In Proceedings of the 2016 CHI Conference on Human Factors in Computing Systems - CHI '16, 2098-2110. DOI:https://doi.org/10.1145/2858036.2858207

[7] Martin. A. Conway. 2001. Sensory-perceptual episodic memory and its context: Autobiographical memory. In Philosophical Transactions of the Royal Society B: Biological Sciences, 1375-1384. DOI:https://doi.org/10.1098/rstb.2001.0940

[8] Martin A Conway. 2005. Memory and the self. Fournal of Memory and Language 53, 594-628. DOI:https://doi.org/10.1016/j.jml.2005.08.005

[9] David Coyle and Gavin Doherty. 2009. Clinical Evaluations and Collaborative Design: Developing New Technologies for Mental Healthcare Interventions. Proc. SIGCHI Conf. Hum. Factors Comput. $\begin{array}{llll}\text { Syst. } & \text { CHI'09 2051-2060 }\end{array}$ DOI:https://doi.org/10.1145/1518701.1519013 
[10] David Coyle, Gavin Doherty, Mark Matthews, and John Sharry. 2007. Computers in talk-based mental health interventions. Interact. Comput. 19, $4 \quad$ (2007), 545-562. DOI:https://doi.org/10.1016/j.intcom.2007.02.001

[11] David Coyle, Nicola McGlade, Gavin Doherty, and Gary O’Reilly. 2011. Exploratory evaluations of a computer game supporting cognitive behavioural therapy for adolescents. Proc. 2011 Annu. Conf. Hum. factors Comput. Syst. - CHI '11 (2011), 2937-2946. DOI:https://doi.org/10.1145/1978942.1979378

[12] Martín Cammarota Cristiano A.Köhler, André F. Carvalho, Gilberto S. Alves, Roger S. McIntyre, Thomas N. Hyphantis. 2015. AutobiographicalMemory Disturbances in Depression: A Novel Therapeutic Target? Neural Plast. 2015, (2015), 1-14. DOI:https://doi.org/10.1155/2015/759139

[13] Tim Dalgleish and Aliza Werner-Seidler. 2014. Disruptions in autobiographical memory processing in depression and the emergence of memory therapeutics. Trends Cogn. Sci. 18, 11 (2014), 596-604. DOI:https://doi.org/10.1016/j.tics.2014.06.010

[14] Claudia Daudén Roquet and Corina Sas. 2018. Evaluating Mindfulness Meditation Apps. In Extended Abstracts of the 2018 CHI Conference on Human Factors in Computing Systems - CHI '18, 1-6. DOI:https://doi.org/10.1145/3170427.3188616

[15] Karen F. Dearing and Ian H. Gotlib. 2009. Interpretation of ambiguous information in girls at risk for depression. 7. Abnorm. Child Psychol. $\quad 37, \quad 1 \quad$ (2009), $79-91$. DOI:https://doi.org/10.1007/s10802-008-9259-z

[16] Lina Dib, Daniela Petrelli, and Steve Whittaker. 2010. Sonic souvenirs: exploring the paradoxes of recorded sound for family remembering. Proc. 2010 ACM Conf. Comput. Support. Coop. Work. CSCW'10 (2010), 391-400. DOI:https://doi.org/http://doi.acm.org/10.1145/1718918.1718985

[17] Gavin Doherty, David Coyle, and John Sharry. 2012. Engagement with online mental health interventions. Proc. 2012 ACM Annu. Conf. Hum. Factors Comput. Syst. - CHI '12 (2012), 1421-1430. DOI:https://doi.org/10.1145/2207676.2208602

[18] Kevin Doherty and Gavin Doherty. 2018. The construal of experience in HCI: Understanding self-reports. Int. F. Hum. Comput. Stud. 110, (2018), 63-74. DOI:https://doi.org/10.1016/j.ijhcs.2017.10.006

[19] Edward R. Watkins, Rod Taylor, Richard Byng, Celine B. Baeyens, Rebecca Read, Katherine A. Pearson. 2012. Guided self-help concreteness training as an intervention for major depression in primary care: a Phase II randomized controlled trial. Psychol. Med. 42, $07 \quad$ (2012), 1359-1371. DOI:https://doi.org/10.1017/S0033291711002480

[20] Gunther Eysenbach. 2005. The Law of Attrition. F. Med. INTERNET Res. 7, 1 (2005), 1. DOI:https://doi.org/10.2196/jmir.7.1.e11

[21] Jennifer Fereday and Eimear Muir-Cochrane. 2006. Demonstrating Rigor Using Thematic Analysis: A Hybrid Approach of Inductive and Deductive Coding and Theme Development. Int. f. Qual. $\begin{array}{lllll}\text { Methods } & 5, & 1 & \text { (2006), } & 80-92 .\end{array}$ DOI:https://doi.org/10.1177/160940690600500107

[22] Kathleen Kara Fitzpatrick, Alison Darcy, and Molly Vierhile. 2017. Delivering Cognitive Behavior Therapy to Young Adults With Symptoms of Depression and Anxiety Using a Fully Automated Conversational Agent (Woebot): A Randomized Controlled Trial. FMIR Ment. Heal. 4, 2 (2017), e19. DOI:https://doi.org/10.2196/mental.7785

[23] Michael L. Free. 2008. Cognitive Therapy in Groups: Guidelines and Resources for Practice, Second Edition. DOI:https://doi.org/10.1002/9780470713235

[24] Morgan Harvey, Marc Langheinrich, and Geoff Ward. 2015. Remembering through lifelogging: A survey of human memory augmentation. Pervasive Mob. Comput. 27, (2015), 14-26. DOI:https://doi.org/10.1016/j.pmcj.2015.12.002
[25] Caitlin Hitchcock, Aliza Werner-Seidler, Simon E. Blackwell, and Tim Dalgleish. 2017. Autobiographical episodic memory-based training for the treatment of mood, anxiety and stress-related disorders: A systematic review and meta-analysis. Clin. Psychol. Rev. 52, (2017), 92-107. DOI:https://doi.org/10.1016/j.cpr.2016.12.003

[26] Steve Hodges, Emma Berry, and Ken Wood. 2011. SenseCam: A wearable camera that stimulates and rehabilitates autobiographical memory. Memory 19, 7 (2011), 685-696. DOI:https://doi.org/10.1080/09658211.2011.605591

[27] Elise van den Hoven and Berry Eggen. 2008. Informing augmented memory system design through autobiographical memory theory. Pers. Ubiquitous Comput. 12, $6 \quad$ (2008), 433-443. DOI:https://doi.org/10.1007/s00779-007-0177-9

[28] Elise Van Den Hoven, Corina Sas, and Steve Whittaker. 2012. Introduction to this special issue on designing for personal memories: Past, present, and future. Human-Computer Interact. 27, 1-2 (2012), 1-12. DOI:https://doi.org/10.1080/07370024.2012.673451

[29] Sky Tien-yun Huang and Chloe Mun Yee Kwan. 2014. The Moment: a mobile tool for people with depression or bipolar disorder. In International Conference on Ubiquitous ComputingUbiComp '14, 235-238. DOI:https://doi.org/10.1145/2638728.2638784

[30] Nigel Davies. Huy Viet Le, Sarah Clinch, Corina Sas, Tilman Dingler, Niels Henze. 2016. Impact of Video Summary Viewing on Episodic Memory Recall. In Proceedings of the 2016 CHI Conference on Human Factors in Computing Systems - CHI '16, 4793-4805. DOI:https://doi.org/10.1145/2858036.2858413

[31] Ellen Isaacs, Artie Konrad, Alan Walendowski, Thomas Lennig, Victoria Hollis, and Steve Whittaker. 2013. Echoes From the Past: How Technology Mediated Reflection Improves Well-Being. Proc. SIGCHI Conf. Hum. Factors Comput. Syst. -CHI '13 (2013), 10711080. DOI:https://doi.org/bhqm

[32] Marije Kanis and Willem Paul Brinkman. 2010. Making mundane pleasures visible: Mediating daily likings with lightweight technology. Pers. Ubiquitous Comput. 14, 3 (2010), 261-269. DOI:https://doi.org/10.1007/s00779-009-0256-1

[33] Artie Konrad, Simon Tucker, John Crane, and Steve Whittaker. 2016. Technology and Reflection: Mood and Memory Mechanisms for Well-Being. Psychol. Well. Being. 6, 1 (2016), 5. DOI:https://doi.org/10.1186/s13612-016-0045-3

[34] Kees Korrelboom, Martie de Jong, Irma Huijbrechts, and Peter Daansen. 2009. Competitive Memory Training (COMET) for Treating Low Self-Esteem in Patients With Eating Disorders: A Randomized Clinical Trial. f. Consult. Clin. Psychol. 77, 5 (2009), 974-980. DOI:https://doi.org/10.1037/a0016742

[35] Matthew L. Lee and Anind K. Dey. 2007. Capture \& Access Lifelogging Assistive Technology for People with Episodic Memory Impairment Non-technical Solutions. Work. Intell. Syst. Assist. Cogn. (2007), 1-9.

[36] Matthew L. Lee and Anind K. Dey. 2008. Lifelogging memory appliance for people with episodic memory impairment. Proc. 10th Int. Conf. Ubiquitous Comput. - UbiComp '08 (2008), 44-53. DOI:https://doi.org/10.1145/1409635.1409643

[37] Lydia Manikonda and Munmun De Choudhury. 2017. Modeling and Understanding Visual Attributes of Mental Health Disclosures in Social Media. In Proceedings of the 2017 CHI Conference on Human Factors in Computing Systems - CHI '17, 170-181. DOI:https://doi.org/10.1145/3025453.3025932

[38] Mark Matthews and Gavin Doherty. 2011. In the mood: Engaging teenagers in psychotherapy using mobile phones. Chi 2011 (2011), 2947-2956. DOI:https://doi.org/10.1145/1978942.1979379

[39] Kendal L Maxwell and Jennifer Callahan. 2013. MEmory Specificity Training ( MEST ) for Group Treatment of Posttraumatic Stress 
Disorder Therapist's Manual.

[40] John McCarthy and Peter Wright. 2005. Putting "felt-life" at the centre of human-computer interaction (HCI). Cogn. Technol. Work 7, 4 (2005), 262-271. DOI:https://doi.org/10.1007/s10111-005-0011$\mathrm{y}$

[41] Ine Mols, Elise van den Hoven, and Berry Eggen. 2016. Informing Design for Reflection: an Overview of Current Everyday Practices. Proc. 9th Nord. Conf. Human-Computer Interact. - Nord. '16 (2016), 1-10. DOI:https://doi.org/10.1145/2971485.2971494

[42] Heather S. Packer, Ash Smith, and Paul Lewis. 2012. MemoryBook: Generating Narratives from Lifelogs. Proc. 2nd Work. Narrat. $\begin{array}{llllll}\text { hypertext } & - & \text { NHT } & \text { '12 } & \text { (2012), } & 7 .\end{array}$ DOI:https://doi.org/10.1145/2310076.2310079

[43] James W. Pennebaker. 2017. Expressive Writing in Psychological Science. Perspect. Psychol. Sci. 13, 2 (2017), 226-229. DOI:https://doi.org/10.1177/1745691617707315

[44] Daniela Petrelli, Elise van den Hoven, and Steve Whittaker. 2009. Making history: Intentional Capture of Future Memories. Proc. 27th Int. Conf. Hum. factors Comput. Syst. - CHI 09 April (2009), 1723. DOI:https://doi.org/10.1145/1518701.1518966

[45] Chengcheng Qu and Corina Sas. 2018. Exploring Memory Interventions in Depression through Lifelogging Lens. In Proceedings of British HCI 2018, In press.

[46] Filip Raes, J. Mark G. Williams, and Dirk Hermans. 2009. Reducing cognitive vulnerability to depression: A preliminary investigation of MEmory Specificity Training (MEST) in inpatients with depressive symptomatology. F. Behav. Ther. Exp. Psychiatry 40, 1 (2009), 24-38. DOI:https://doi.org/10.1016/j.jbtep.2008.03.001

[47] Stefan Rennick-Egglestone, Sarah Knowles, Gill Toms, Penny Bee, Karina Lovell, and Peter Bower. 2016. Health Technologies "In the Wild": Experiences of Engagement with Computerised CBT. Proc. 2016 CHI Conf. Hum. Factors Comput. Syst. CHI'16 (2016), 21242135. DOI:https://doi.org/10.1145/2858036.2858128

[48] Pedro Sanches, Pavel Karpashevich, Gavin Doherty, Axel Janson, Charles Windlin, Corina Sas, Camille Nadal, and Kristina Höök. 2019. HCI and Affective Health. Taking stock of a decade of studies and charting future research directions. In Conference on Human Factors in Computing Systems -CHI'19, In press.

[49] Corina Sas. 2018. Exploring Self-Defining Memories in Old Age and their Digital Cues. Proc. 2018 Des. Interact. Syst. Conf. DIS'18 June $2018 \quad$ (2018), $149-161$. DOI:https://doi.org/10.1145/3196709.3196767

[50] Corina Sas, Scott Challioner, Christopher Clarke, Ross Wilson, Alina Coman, Sarah Clinch, Mike Harding, and Nigel Davies. 2015. Self-Defining Memory Cues: Creative Expression and Emotional Meaning. Ext. Abstr. ACM CHI'15 Conf. Hum. Factors Comput. Syst. 2, (2015), 2013-2018. DOI:https://doi.org/10.1145/2702613.2732842

[51] Corina Sas, Alan Dix, Nigel Davies, and Adrian Friday. 2006. Capturing and sharing war memories. In In CHI'06: Human factors in computing systems, Workshop on" Designing for collective remembering.

[52] Corina Sas and Matthew Rees. 2013. AffectCam: Arousal -
Augmented SenseCam for Richer Recall of Episodic Memories. (2013), 1041-1046.

[53] Corina Sas, Shuang Ren, Alina Coman, Sarah Clinch, and Nigel Davies. 2016. Life Review in End of Life Care. In Proceedings of the 2016 CHI Conference Extended Abstracts on Human Factors in Computing Systems - CHI EA '16, 2947-2953. DOI:https://doi.org/10.1145/2851581.2892491

[54] Abigail J. Sellen and Steve Whittaker. 2010. Beyond total capture: A Constructive Critique of Lifelogging. Commun. ACM 53, 5 (2010), 70-77. DOI:https://doi.org/10.1145/1735223.1735243

[55] Anna Ståhl, Kristina Höök, Martin Svensson, Alex S. Taylor, and Marco Combetto. 2009. Experiencing the affective diary. Pers. Ubiquitous Comput. 13, 5 (2009), 365-378. DOI:https://doi.org/10.1007/s00779-008-0202-7

[56] Molly M. Stevens, Gregory D. Abowd, Khai N. Truong, and Florian Vollmer. 2003. Getting intothe Living Memory Box: Family archives \& holistic design. Pers. Ubiquitous Comput. 7, 3-4 (2003), 210-216. DOI:https://doi.org/10.1007/s00779-003-0220-4

[57] Emily A. Holmes Tamara J. Lang, Simon E. Blackwell, Catherine J. Harmer, Phil Davison. 2012. Cognitive Bias Modification Using Mental Imagery for Depression: Developing a Novel Computerized Intervention to Change Negative Thinking Styles. Personal. Inf. Process. 26, 2 (2012), 87-189. DOI:https://doi.org/10.1002/per

[58] Sho Tsugawa, Yusuke Kikuchi, Fumio Kishino, Kosuke Nakajima, Yuichi Itoh, and Hiroyuki Ohsaki. 2015. Recognizing Depression from Twitter Activity. Proc. 33rd Annu. ACM Conf. Hum. Factors Comput. Syst. - CHI '15 (2015), 3187-3196. DOI:https://doi.org/10.1145/2702123.2702280

[59] L. A. Watson, D. Berntsen, W. Kuyken, and E. R. Watkins. 2013. Involuntary and voluntary autobiographical memory specificity as a function of depression. F. Behav. Ther. Exp. Psychiatry 44, 1 (2013), 7-13. DOI:https://doi.org/10.1016/j.jbtep.2012.06.001

[60] J Mark G Williams, Thorsten Barnhofer, Catherine Crane, Dirk Herman, Filip Raes, Ed Watkins, and Tim Dalgleish. 2007. Autobiographical memory specificity and emotional disorder. Psychol. Bull. 133, 1 (January 2007), 122-48. DOI:https://doi.org/10.1037/0033-2909.133.1.122

[61] Naomi Yamashita, Hideaki Kuzuoka, Keiji Hirata, Takashi Kudo, Eiji Aramaki, and Kazuki Hattori. 2017. Changing Moods: How Manual Tracking by Family Caregivers Improves Caring and Family Communication. Proc. SIGCHI Conf. Hum. Factors Comput. $\begin{array}{llll}\text { Syst. } & -\mathrm{CHI} & \text { '17 } & \text { (2017), }\end{array}$ DOI:https://doi.org/10.1145/3025453.3025843

[62] Extract from the CBT and Feeling Good. Retrieved September 21, 2018 from

https://iveronicawalsh.files.wordpress.com/2012/06/cbtafg_abcdex tract_handout.pdf

[63] Using the Feelings Wheel $\mid$ Art It Out Therapy Center | Therapy | Atlanta | East Cobb | Buckhead. Retrieved September 21, 2018 from https://www.artitout.com/single-post/2014/12/04/Using-theFeelings-Wheel 